\title{
Parallel Correlative Study of the Heat Balance Test Process for Testing the Efficiency of Turbo-Generators
}

\author{
Luqman Muhammed Audu1, Olugbenga Olanrewaju Noah², Ogaga Kenneth Ajaino3, \\ Friday Chukwuyem Igbesi ${ }^{4}$ \\ ${ }^{1}$ Department of Mechanical Engineering, Auchi Polytechnic, Auchi, Nigeria \\ ${ }^{2}$ Department of Mechanical Engineering, University of Lagos, Lagos, Nigeria \\ ${ }^{3}$ Department of Mechanical Engineering, Delta State Polytechnic, Ogwashuku, Nigeria \\ ${ }^{4}$ Department of Mechanical Engineering, Delta State Polytechnic, Otefe-Oghara, Nigeria \\ Email: luqslama@gmail.com, onoah@unilag.edu.ng
}

Received 25 January 2016; accepted 22 March 2016; published 25 March 2016

Copyright (C) 2016 by authors and Scientific Research Publishing Inc.

This work is licensed under the Creative Commons Attribution International License (CC BY).

http://creativecommons.org/licenses/by/4.0/

(c) (i) Open Access

\begin{abstract}
In order to prolong the life span of a turbo-generator plant and sustain its performance at high efficiency, it is subjected periodically to regular test to monitor the operational profile and efficiency of power conversion from mechanical energy to electrical energy. Analysis of these test data serves as a measure to indicate deviation from normal operation profile and deterioration of plant performance. This present work implemented the heat balance tests process to three turbogenerator units in order to assess the harmony, consistency, and accuracy of results to establish parallel correlation for the test process. The test process involves carrying out a heat balance for the turbo-generators at $50 \%, 75 \%$ and $100 \%$ load respectively through the determination of the heat losses through the hydrogen coolers, bearing oil, seal oil and radiation and convention to the atmosphere. Some important results were presented in the paper.
\end{abstract}

\section{Keywords}

Efficiency, Turbo-Generator, Testing, Hydrogen-Cooled, Heat Balance

\section{Introduction}

Turbo-generators are usually used to produce electricity by using a turbine to drive an alternative current (a.c) generator. The choice of machinery for high power generation currently comes down to either hydrogen cooled 
generators providing high efficiency or air cooled generator characterized by easy operation and maintenance at low cost [1]. Consequently, the world powered engineering has experienced an increase in the demand for high powered turbo-generators with hydrogen and air cooling [2], for the generation of electric power for the sustenance of vital national production processes and services.

Turbo-generators convert mechanical energy of rotation of the turbine shaft into electricity. The a.c generator consists principally of a magnetic circuit, direct-current (d.c) field winding, a-c armature winding and a mechanical structure including a cooling and lubricating system. The magnetic circuit and field winding are arranged so that as the machine rotates, the magnetic flux linking the armature windings change cyclically, thereby inducing alternating voltage in the armature winding.

Heat is produced in a generator as a result of resistive losses caused by current flow in the stator and field windings, stator core magnetic losses and windage losses. This heat generation if not controlled and regulated through a cooling system can cause breakdown in insulation and other damages to vital component of the machine. By providing forced cooling of the rotating and stationary components, the generator ratings may be increased and the physical components may be made smaller [3]. To ensure reliable operation of turbo-generators, the heating of any electrical part of the machine must be kept within certain limits [4].

Turbo-generators were designed exclusively for air-cooling in the early stages of development. Hydrogen gas was later used as the cooling medium for turbo-generators of high rating, although recent development of a high performance and high efficiency inner air cooling ventilation system generator with rating of 250 MVA has been achieved [1]. This generator was upgraded by the optimization of the stator core duct and ventilation structure, stator coil structure, and the modification of the ventilation structure of the rotor end, pushing the efficiency to $98.80 \%$ which is close to that of hydrogen cooled generators [5]. Further modifications were made to significantly reduce the noise level of the turbo-generator [6]; this development put air cooled turbo-generators at par with their hydrogen counterpart in terms of power ratings. Figure 1 represents the schematic of cooling method for inner cooler cooling system. Air cooled turbo-generators, in general, tend to generate more noise compared to hydrogen-cooled generators.

Inspection, testing and regular checks are vital aspects of maintenance procedures that assist in sustaining the operation, performance of the plants and provide appropriate information for timely maintenance to provide the needed availability and reliability for plant operation and satisfactory performance [7]. The following research works on turbo-generator's operation and performance have been carried out. [8] studied the temperature generation of the elementary conductors of the stator windings of a $225 \mathrm{MW}$ turbo-generator with fiber optics sensors. Suitable parameters for the design specification of the thermal conductivity and heat transfer coefficient of air cooled turbo-generators were obtained and characterized. [5] discussed the development of $250 \mathrm{MVA}, 60 \mathrm{HZ}$ air cooled generator with the optimization of the ventilation structure and stator coil strand arrangements. The generator's performance achieved a high efficiency of $98.80 \%$ with output characteristics that competes with hydrogen cooled generators. [9] investigated the air gap ventilation, stator strand and rotor winding temperature

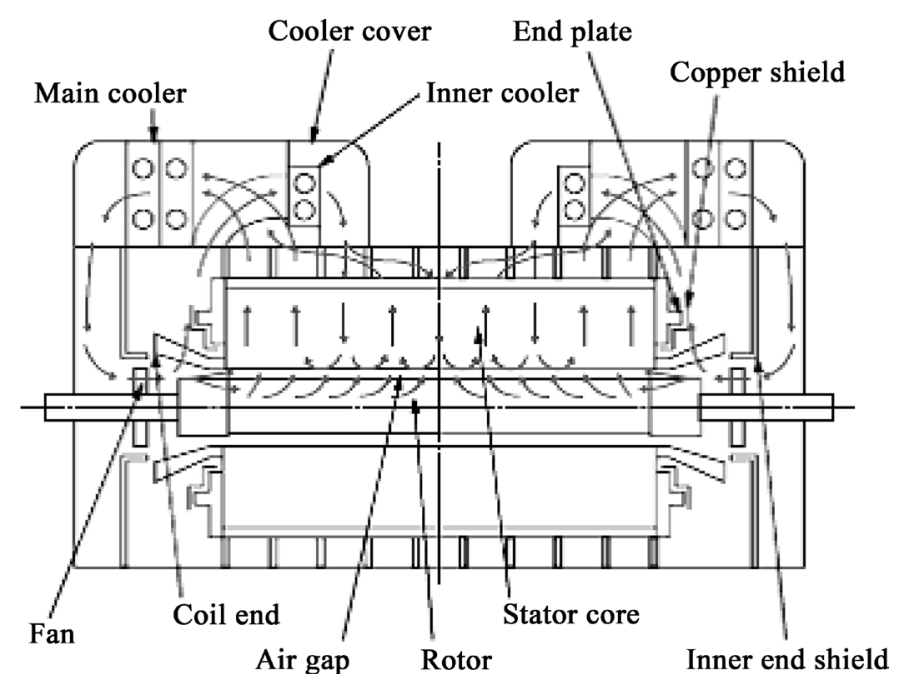

Figure 1. Schematic of cooling method for Inner cooler cooling system. 
distribution in a 250 MVA air cooled turbo-generator. The temperature measured under full load conditions was under the limits of class B insulation, while the measured air flow and temperature distribution in the stator, rotor and air gap conformed to the values obtained by the system design programs. [10] evaluated the reliability of a 250 MVA generator by connection to a high capacity load machine with the application of a rated field current to reproduce field conditions. The maximum temperature rise was observed to be accommodated between the limits of class B insulation. [1] evaluated the performance of a $250 \mathrm{MVA}(50 / 60 \mathrm{~Hz})$ large air cooled turbogenerator with inner cooler ventilation system. The results obtained were used to develop a database for the design and construction of hydrogen and air cooled turbo-generator with class B temperature rise standard.

Two general types of test are carried out on turbo-generator in thermal power stations. These are the guaranteed performance test or acceptance test and routine test. The guaranteed performance test is carried out immediately after commissioning to ensure that the performance of the machine meets the specification given by the manufacturer. This performance test is carried out using the Work Test Process. The routine test is carried out during normal operation of the machine at regular interval to ascertain the generator performance and to provide information on generator efficiency, causes of depreciation in generator performance and an effective guide to maintenance of the generators.

The Work Test Process is carried out after generator has attained operational stability. In this process six losses are evaluated for the generator. Two of the losses are considered to be fixed losses. They are fixed with input to load for the generator. The fixed losses are the windage and friction losses and core loss. The rest losses are variable and they include field copper (rotor) losses, armature copper loss, stray load loss and excitation loss [11].

The Work Test Process could not be used to carry out the routine test at Egbin Thermal Station because it entails disengaging the turbo-generator from the national power grid which will result to the loss of at least 220 MW out of an actual national power output that fluctuates between 2600 MW - 3600 MW per day [12]. Statistics from the Federal Ministry of Energy indicates the peak power generation as at October, 2014 to be 3513.5 MW [13]. Therefore, in the face of the present power predicament there is the dire need to develop a routine test process for evaluating the efficiency of turbo-generators without disrupting their operation and power generation to the power grid by the Power Holding Company of Nigeria (PHCN). This is achieved by carrying out a heat balance on the turbo-generator system. The heat balance test process had earlier been used to evaluate the efficiency of one turbo-generator unit at Egbin Thermal Station [14]. In other to establish parallel correlation for the heat balance test process, there is the essential need to extend the test process to three turbo-generator units to assess the harmony and consistency of results between the three units.

The objective of the study is the implementation of the heat balance testing process to three turbo-generator units in order to assess the harmony, consistency and accuracy of results to establish parallel correlation for the test process. The results will also be used to determine if there has been any significant deterioration in the efficiency of the plant by a comparative analysis with the results of the work test process used to commission the plants.

\section{Methodology}

A heat balance for the turbo-generator plant was carried out by taking readings on embedded and supervisory equipment on the turbo-generator on $100 \%, 75 \%$ and $50 \%$ operational mode respectively. The reading were used to calculate the heat losses though the hydrogen coolers, bearing oil, shaft seal oil and by radiation and convention through the generator hood to the atmosphere.

The summary of the process is as follows:

1) Reading of the mass flow rate and temperature elevation of the flow of hydrogen through the rotor.

2) Reading of the water flow rate and temperature elevation of the flow of water through the stator.

3) Reading of the mass flow rate and temperature elevation of the bearing oil.

4) Reading of the mass flow rate and temperature elevation of the shaft seal oil.

5) Measuring of the heat losses through radiation and convention to the atmosphere through the generator hood.

The result of the Heat Balance Test Process was compared to that of the Work Test Process to establish the reliability of the test results using the Hitachi result as a reference point. The result was also used to deduce if there has been any significant deterioration in the efficiency of the plants.

\subsection{Temperature Measurement of Generator Hood Surface}

The temperature of the generator hood was measured by an infra-red thermometer to determine the loss of heat 
by radiation and convention to the atmosphere through the generator's hood surface. The generator hood which is semi-circular in shape was divided into nineteen equal parts and the temperature taken at each point. The average temperature was then taken for the generator at the various loads.

\subsection{Procedure for Calculating Heat Losses}

1) The Heat losses in hydrogen coolers

a) Heat losses in hydrogen cooler $A=Q_{1}=M_{W A} \times C P_{W A} \times \theta_{W A}(\mathrm{~kW})$

b) Heat losses in hydrogen cooler $B=Q_{2}=M_{W B} \times C P_{W B} \times \theta_{W B}(\mathrm{~kW})$

c) Heat losses in hydrogen cooler $C=Q_{3}=M_{W C} \times C P_{W C} \times \theta_{W C}(\mathrm{~kW})$

d) Heat losses in hydrogen cooler $D=Q_{4}=M_{W D} \times C P_{W D} \times \theta_{W D}(\mathrm{~kW})$

where $M_{W}$ is mass flow rate $(\mathrm{kg} / \mathrm{s})$ of water through the cooler, $C P_{W}$ is the specific heat capacity of water $(\mathrm{kJ} / \mathrm{kg} \cdot \mathrm{K}), \theta_{W} \mathbb{F}$ is the temperature difference across the cooler $\left({ }^{\circ} \mathrm{C}\right)$.

2) Heat losses through bearing oil

a) Heat losses in bearing oil (turbine side)

$$
Q_{5}=M_{T} \times C P \times \theta_{T}(\mathrm{~kW})
$$

b) Heat losses in bearing oil (collector ring side) $Q_{6}=M_{C} \times C P_{T} \times \theta_{c}(\mathrm{~kW})$

3) Heat losses through shaft seal oil (turbine Side) $Q_{7}=M_{S T} \times C P_{T} \times \theta_{\text {st }}(\mathrm{kW})$

Heat losses through seal oil (collector ring side) $Q_{8}=M_{S C} \times C P_{T} \times \theta_{s c}(\mathrm{~kW})$

where $M_{T}, M_{C}$, are the mass flow rate $(\mathrm{kg} / \mathrm{s})$ of bearing oil at the turbine and the collector ring side respectively, $M_{S T}, M_{S C}$ are the mass flow rate $(\mathrm{kg} / \mathrm{s})$ of seal oil at the turbine and collector ring side respectively.

4) Heat losses by convention and radiation $Q_{9}=h \times A \times\left(t_{s}-t_{a}\right)(\mathrm{kW})$ where $h$ is the combined coefficient of heat transfer from generator surface, $A$ is the area of generator surface in contact with air $\left(\mathrm{m}^{2}\right), t_{s}$ is the generator surface temperature $\left({ }^{\circ} \mathrm{C}\right), t_{a}$ is the ambient air temperature $\left({ }^{\circ} \mathrm{C}\right)$.

Generator shaft power input $=$ power generated + total losses

$$
\text { Efficiency }=\frac{\text { Power generated } \times 100}{\text { Power input }}
$$

\section{Test Data}

\subsection{Balance Test Data}

Various readings taken from the turbo-generator supervisory and monitoring equipment areindicated in the tables below (see Tables 1-6) for the various operating conditions. The readings include flow rates and temperature readings for bearing oil, seal oil and water flow in hydrogen coolers.

Unit I (Turbo-Generating Set 1)

Unit 4 (Turbo-Generating Set 4)

Unit 6 (Turbo-Generating Set 6)

\subsection{Guaranteed Test Data}

Table 5 indicates the calculated losses for the guaranteed test at the operating temperature for generator operating load condition of 221.2 MW at Maximum Continuous Rating (MCR), which is approximately $100 \%$ load.

\subsection{Material Properties}

1) Specific heat capacity of water at the mean temperature $=4.179 \mathrm{~kJ} / \mathrm{kg} \cdot \mathrm{K}$.

2) Specific heat capacity of bearing oil $=1.881 \mathrm{~kJ} / \mathrm{kg} \cdot \mathrm{K}$.

3) Combined coefficient of heat transfer from generator surface to air $=12.40 \mathrm{~W} / \mathrm{m}^{2}$.

4) Area of contact of generator surface with air $=60.7085 \mathrm{~m}^{2}$.

5) Ambient air temperature $=29^{\circ} \mathrm{C}$. 
Table 1. Flow rates and temperature reading on unit 1.

\begin{tabular}{|c|c|c|c|c|c|}
\hline \multirow[t]{2}{*}{$\mathbf{S} / \mathbf{N}$} & \multirow[t]{2}{*}{ Item } & \multirow{2}{*}{$\begin{array}{l}\text { Unit } \\
\text { MW }\end{array}$} & \multicolumn{3}{|c|}{ Operation condition } \\
\hline & & & 219 & 163 & 111 \\
\hline i. & Bearing oil inlet temp. & ${ }^{\circ} \mathrm{C}$ & 47.50 & 47.50 & 47.00 \\
\hline \multirow[t]{2}{*}{ ii. } & Bearing oil outlet temp & ${ }^{\circ} \mathrm{C}$ & 5 & & \\
\hline & Turbine side (TBS) & ${ }^{\circ} \mathrm{C}$ & 54.80 & 54.00 & 53.00 \\
\hline iii. & Collector ring sides (CLRS) & ${ }^{\circ} \mathrm{C}$ & 54.00 & 53.50 & 52.50 \\
\hline iv & Bearing oil flow rate (TBS) & $\mathrm{kg} / \mathrm{s}$ & 3.83 & 3.20 & 2.62 \\
\hline iv & Bearing out flow rate (CLRS) & $\mathrm{kg} / \mathrm{s}$ & 3.83 & 3.20 & 2.62 \\
\hline B & Hydrogen coolers (water flow rate) & $\mathrm{kg} / \mathrm{s}$ & & & \\
\hline i. & Cooler A & $\mathrm{kg} / \mathrm{s}$ & 22.22 & 19.03 & 15.57 \\
\hline Ii & Cooler B & $\mathrm{kg} / \mathrm{s}$ & 22.22 & 19.02 & 15.56 \\
\hline iii. & Cooler C & $\mathrm{kg} / \mathrm{s}$ & 22.22 & 19.02 & 15.56 \\
\hline iv. & Cooler D & $\mathrm{kg} / \mathrm{s}$ & 22.20 & 19.00 & 15.43 \\
\hline $\mathrm{v}$ & Water inlet temp. (Cooler A-D) & $\mathrm{kg} / \mathrm{s}$ & 34 & 34 & 34 \\
\hline \multirow[t]{5}{*}{ vi } & Water outlet temp. & ${ }^{\circ} \mathrm{C}$ & & & \\
\hline & Cooler A & ${ }^{\circ} \mathrm{C}$ & 42.30 & 41.50 & 41.00 \\
\hline & Cooler B & ${ }^{\circ} \mathrm{C}$ & 41.00 & 40.00 & 39.50 \\
\hline & Cooler C & ${ }^{\circ} \mathrm{C}$ & 42.00 & 41.00 & 40.50 \\
\hline & Cooler D & ${ }^{\circ} \mathrm{C}$ & 42.00 & 41.34 & 40.50 \\
\hline $\mathrm{C}$ & Shaft seal oil & ${ }^{\circ} \mathrm{C}$ & & & \\
\hline $\mathrm{i}$ & Seal oil inlet temp. & ${ }^{\circ} \mathrm{C}$ & 44.00 & 44:00 & 44.00 \\
\hline ii & Seal oil outlet temp (TBS) & ${ }^{\circ} \mathrm{C}$ & 66.00 & 63.50 & 61.50 \\
\hline iii & Seal oil outlet temp(CLRS) & ${ }^{\circ} \mathrm{C}$ & 64.50 & 62.00 & 60.50 \\
\hline iv & Seal oil flow rate (TBS) & $\mathrm{kg} / \mathrm{s}$ & 1.12 & 0.972 & 0.764 \\
\hline $\mathrm{v}$ & Seal oil flow rate (CLRS) & $\mathrm{kg} / \mathrm{s}$ & 1.11 & 0.972 & 0.764 \\
\hline $\mathrm{T}_{\mathrm{M}}$ & Mean generator surface temperature & ${ }^{\circ} \mathrm{C}$ & 54 & 53 & 49 \\
\hline
\end{tabular}

Table 2. Flow rates and temperature reading on unit 4.

\begin{tabular}{|c|c|c|c|c|c|}
\hline & \multirow[t]{2}{*}{ Item } & \multirow{2}{*}{$\begin{array}{l}\text { Unit } \\
\text { MW }\end{array}$} & \multicolumn{3}{|c|}{ Operation condition } \\
\hline & & & 219 & 164 & 110 \\
\hline A & Bearing oil & & & & \\
\hline $\mathrm{i}$ & Bearing oil inlet temp & ${ }^{\circ} \mathrm{C}$ & 47.00 & 47.00 & 47.00 \\
\hline ii & Bearing oil outlet temp (CLRS) & ${ }^{\circ} \mathrm{C}$ & 54.50 & 54.00 & 52.00 \\
\hline iii & Bearing oil outlet temp (TBS) & ${ }^{\circ} \mathrm{C}$ & 56.00 & 55.00 & 53.00 \\
\hline iv & Bearing oil flow rate (TBS) & $\mathrm{kg} / \mathrm{s}$ & 3.83 & 3.20 & 2.62 \\
\hline $\mathrm{V}$ & Bearing oil flow rate (CLRS) & $\mathrm{kg} / \mathrm{s}$ & 3.83 & 3.20 & 2.62 \\
\hline $\mathrm{B}$ & & & & & \\
\hline $\mathrm{i}$ & $\begin{array}{l}\text { Water flow rates } \\
\text { Cooler A } \\
\text { Cooler B } \\
\text { Cooler C } \\
\text { Cooler D }\end{array}$ & $\begin{array}{l}\mathrm{kg} / \mathrm{s} \\
\mathrm{kg} / \mathrm{s} \\
\mathrm{kg} / \mathrm{s} \\
\mathrm{kg} / \mathrm{s} \\
\mathrm{kg} / \mathrm{s}\end{array}$ & $\begin{array}{l}22.21 \\
22.21 \\
22.22 \\
22.21\end{array}$ & $\begin{array}{l}19.04 \\
19.02 \\
19.03 \\
19.01\end{array}$ & $\begin{array}{l}15.55 \\
15.54 \\
15.56 \\
15.47\end{array}$ \\
\hline ii & $\begin{array}{l}\text { Water inlet temperature } \\
\text { Cooler A-D }\end{array}$ & ${ }^{\circ} \mathrm{C}$ & 34 & 34 & 34 \\
\hline iii & $\begin{array}{l}\text { Water outlet temp } \\
\text { Cooler A } \\
\text { Cooler B } \\
\text { Cooler C } \\
\text { Cooler D }\end{array}$ & $\begin{array}{l}{ }^{\circ} \mathrm{C} \\
{ }^{\circ} \mathrm{C} \\
{ }^{\circ} \mathrm{C} \\
{ }^{\circ} \mathrm{C}\end{array}$ & $\begin{array}{l}42.50 \\
41.50 \\
42.50 \\
41.50\end{array}$ & $\begin{array}{l}40.00 \\
40.00 \\
41.00 \\
41.00\end{array}$ & $\begin{array}{l}40.00 \\
39.00 \\
40.50 \\
40.00\end{array}$ \\
\hline
\end{tabular}




\section{Continued}

\begin{tabular}{rllcll}
\hline C & Shaft seal oil & & & & \\
i & Seal oil inlet temperature & ${ }^{\circ} \mathrm{C}$ & 43.50 & 44.50 & 44.00 \\
ii & Seal oil outlet temp (TBS) & ${ }^{\circ} \mathrm{C}$ & 66.00 & 64.00 & 62.50 \\
iii & Seal oil outlet temp (CLRS) & ${ }^{\circ} \mathrm{C}$ & 65.00 & 63.00 & 61.50 \\
iv & Seal oil flow rate (TBS) & $\mathrm{kg} / \mathrm{s}$ & 1.13 & 0.975 & 0.764 \\
v & Seal flow rate (CLRS) & $\mathrm{kg} / \mathrm{s}$ & 1.12 & 0.975 & 0.764 \\
$\mathrm{~T}_{\mathrm{M}}$ & Mean generator surface temperature & ${ }^{\circ} \mathrm{C}$ & 53.00 & 50.00 & 47.85 \\
\hline
\end{tabular}

Table 3. Flow rates and temperature reading for unit 6 .

\begin{tabular}{|c|c|c|c|c|c|}
\hline & \multirow[t]{2}{*}{ Item } & \multirow{2}{*}{$\begin{array}{l}\text { Unit } \\
\text { MW }\end{array}$} & \multicolumn{3}{|c|}{ Operation condition } \\
\hline & & & 220 & 166 & 110 \\
\hline A & Bearing oil & & & & \\
\hline $\mathrm{i}$ & Bearing oil inlet temperature & ${ }^{\circ} \mathrm{C}$ & 48.00 & 48.00 & 48.50 \\
\hline ii & Bearing oil outlet temperature (TBS) & ${ }^{\circ} \mathrm{C}$ & 56.50 & 55.00 & 53.50 \\
\hline iii & Bearing oil outlet temperature (CLRS) & ${ }^{\circ} \mathrm{C}$ & 55.50 & 54.00 & 52.50 \\
\hline iv & Bearing oil flowrate (TBS) & $\mathrm{kg} / \mathrm{s}$ & 3.84 & 3.21 & 2.63 \\
\hline $\mathrm{v}$ & Bearing oil flowrate (CLRS) & $\mathrm{kg} / \mathrm{s}$ & 3.84 & 3.21 & 2.63 \\
\hline \multicolumn{6}{|l|}{ B } \\
\hline \multirow{5}{*}{$\mathrm{i}$} & Water flow rates & $\mathrm{kg} / \mathrm{s}$ & & & \\
\hline & Cooler A & $\mathrm{kg} / \mathrm{s}$ & 22.24 & 19.05 & 15.44 \\
\hline & Cooler B & $\mathrm{kg} / \mathrm{s}$ & 22.24 & 19.04 & 15.45 \\
\hline & Cooler C & $\mathrm{kg} / \mathrm{s}$ & 22.24 & 19.05 & 15.48 \\
\hline & Cooler D & $\mathrm{kg} / \mathrm{s}$ & 22.24 & 19.01 & 15.20 \\
\hline ii & Water inlet temperature A-D & ${ }^{\circ} \mathrm{C}$ & 34.00 & 34.00 & 34.00 \\
\hline \multirow{5}{*}{ iii } & Water outlet temp. & & & & \\
\hline & Cooler A & ${ }^{\circ} \mathrm{C}$ & 42.00 & 41.00 & 40.50 \\
\hline & Cooler B & ${ }^{\circ} \mathrm{C}$ & 42.00 & 41.50 & 40.50 \\
\hline & Cooler C & ${ }^{\circ} \mathrm{C}$ & 43.00 & 41.00 & 40.00 \\
\hline & Cooler D & ${ }^{\circ} \mathrm{C}$ & 42.50 & 41.00 & 40.00 \\
\hline $\mathrm{C}$ & Shaft seal oil & & & & \\
\hline $\mathrm{i}$ & Seal oil inlet temperature & ${ }^{\circ} \mathrm{C}$ & 44.50 & 44.00 & 44.00 \\
\hline ii & Seal oil outlet temp (TBS) & ${ }^{\circ} \mathrm{C}$ & 66.50 & 64.00 & 61.50 \\
\hline iii & Seal oil outlet temp (CLRS) & ${ }^{\circ} \mathrm{C}$ & 65.00 & 63.50 & 60.00 \\
\hline iv & Seal oil flowrate (TBS) & $\mathrm{kg} / \mathrm{s}$ & 1.103 & 0.981 & 0.792 \\
\hline $\mathrm{v}$ & Seal flowrate (CLRS) & $\mathrm{kg} / \mathrm{s}$ & 1.100 & 0.981 & 0.792 \\
\hline $\mathrm{T}_{\mathrm{M}}$ & $\begin{array}{l}\text { Mean generator surface } \\
\text { Temperature }\end{array}$ & ${ }^{\circ} \mathrm{C}$ & 53.00 & 50.47 & 47.21 \\
\hline
\end{tabular}

Table 4. Total water flow rates for unit 1 , unit 4 and unit 6.

\begin{tabular}{|cccc}
\hline \multirow{2}{*}{ Load } & & Total water flowrates (kg/s) & Unit 6 \\
\cline { 2 - 4 } & Unit 1 & Unit 4 & 61.57 \\
$75 \%$ & 62.12 & 62.12 & 76.15 \\
$100 \%$ & 76.07 & 76.10 & 88.96 \\
\hline
\end{tabular}




\subsection{Heat Balance}

Tables 7-9 indicate the heat losses through the bearing oil, seal oil, hydrogen coolers and losses through radiation and convention to the atmosphere. The operating conditions of the turbo-generators are also indicated in percentage of their installed capacity to specify their output in correlation with the losses from the plants.

The efficiencies of the plant at various rated condition are also indicated.

Table 5. Generator efficiency at rated conditions.

\begin{tabular}{lcc}
\hline \multicolumn{1}{c}{ Description } & Operating temp $\left({ }^{\circ} \mathbf{C}\right)$ & Power loss $(\mathbf{k W})$ \\
\hline 1. Armature copper loss & 95 & 329 \\
2. Rotor copper loss & 95 & 758 \\
3. Core loss & & 501 \\
4. Stray load loss & & 665 \\
5. Windage and friction loss at rated hydrogen pressure & 502 \\
6. Excitation loss & & 76 \\
7. Total generator loss & & 2831 \\
8. Efficiency (\%) & 98.73 \\
\hline
\end{tabular}

Source: Egbin Thermal Station Operation Manual by PHCN [15].

Table 6. Summary of generator losses and efficiency.

\begin{tabular}{lcccccc}
\hline & Unit & & & & \\
\hline 1. Operating condition (MCR) & $\%$ & 100 & 75 & 50 & 25 \\
2. Total generator loss & KW & 2831 & 2156.3 & 1629.8 & 1289.0 \\
3. Generator efficiency & $\%$ & 98.73 & 98.71 & 98.54 & 97.71 \\
\hline
\end{tabular}

Table 7. Heat balance for unit 1 .

\begin{tabular}{|c|c|c|c|c|c|}
\hline \multirow[b]{2}{*}{1} & \multirow[t]{2}{*}{ Load } & \multirow{2}{*}{$\begin{array}{l}\text { Unit } \\
\text { MW }\end{array}$} & \multicolumn{3}{|c|}{ Operating conditions (losses) } \\
\hline & & & 219 & 163 & 111 \\
\hline & Load & $\%$ & 99.55 & 74.09 & 50.45 \\
\hline A & Bearing oil losses & & & & \\
\hline I & Turbine side (TBS) & $\mathrm{kW}$ & 57.63 & 39.125 & 29.569 \\
\hline \multirow[t]{2}{*}{ II } & Collector ring side(CLRS) & $\mathrm{kW}$ & 46.83 & 36.115 & 27.105 \\
\hline & Total bearing oil losses & $\mathrm{kW}$ & 104.46 & 75.240 & 56.674 \\
\hline B & Losses through $\mathrm{H}_{2}$ coolers & & & & \\
\hline I & Cooler A & $\mathrm{kW}$ & 770.72 & 596.448 & 455.469 \\
\hline II & Cooler B & $\mathrm{kW}$ & 649.710 & 476.907 & 357.639 \\
\hline III & Cooler C & $\mathrm{kW}$ & 789.290 & 556.392 & 422.664 \\
\hline \multirow[t]{3}{*}{ IV } & Cooler D & $\mathrm{kW}$ & 742.190 & 555.807 & 419.133 \\
\hline & Total cooler losses & $\mathrm{kW}$ & 2951.910 & 2185.554 & 1654.905 \\
\hline & Losses through seal oil & & & & \\
\hline \multirow{3}{*}{$\mathrm{C}$} & Turbine side (TBS) & $\mathrm{kW}$ & 46.350 & 35.652 & 25.149 \\
\hline & Collector ring side (CLRS) & $\mathrm{kW}$ & 42.800 & 32.910 & 23.719 \\
\hline & Total seal oil losses & $\mathrm{kW}$ & 89.150 & 68.562 & 48.868 \\
\hline $\mathrm{D}$ & Losses by radiation and convention & $\mathrm{kW}$ & 18.82 & 18.069 & 15.075 \\
\hline $\mathrm{E}$ & Total losses & $\mathrm{kW}$ & 3164.34 & 2308.335 & 1777.522 \\
\hline $\mathrm{F}$ & Turbo-generator shaft power input & & $222,164.34$ & $165,308.335$ & $112,775.522$ \\
\hline G & Generator efficiency & $\%$ & 98.60 & 98.60 & 98.42 \\
\hline
\end{tabular}


Table 8. Heat balance for unit 4 .

\begin{tabular}{|c|c|c|c|c|c|}
\hline \multirow{2}{*}{$\begin{array}{c}\mathrm{S} / \mathrm{N} \\
1\end{array}$} & \multirow[t]{2}{*}{ Items } & \multirow{2}{*}{$\begin{array}{l}\text { Unit } \\
\text { MW }\end{array}$} & \multicolumn{3}{|c|}{ Operating conditions (losses) } \\
\hline & & & 219 & 164 & 110 \\
\hline & Load & $\%$ & 99.55 & 74.54 & 50 \\
\hline A & Bearing oil losses & & & & \\
\hline $\mathrm{i}$ & Turbine side (TBS) & $\mathrm{kW}$ & 64.838 & 64.838 & 29.56 \\
\hline \multirow[t]{2}{*}{ ii } & Collector ring side (CLRS) & $\mathrm{kW}$ & 54.032 & 54.032 & 24.641 \\
\hline & Total bearing oil losses & $\mathrm{kW}$ & 118.87 & 118.870 & 54.210 \\
\hline B & Losses through $\mathrm{H}_{2}$ coolers & & & & \\
\hline $\mathrm{i}$ & Cooler A & $\mathrm{kW}$ & 788.933 & 596.761 & 389.901 \\
\hline ii & Cooler B & $\mathrm{kW}$ & 696.117 & 596.134 & 324.708 \\
\hline iii & Cooler C & $\mathrm{kW}$ & 789.286 & 596.444 & 390.151 \\
\hline iv & Cooler D & $\mathrm{kW}$ & 696.117 & 556.100 & 387.895 \\
\hline $\mathrm{v}$ & Total cooler losses & $\mathrm{kW}$ & 2970.453 & 2345.442 & 1492.656 \\
\hline $\mathrm{C}$ & Losses through seal oil & & & & \\
\hline $\mathrm{i}$ & Turbine side (TBS) & $\mathrm{kW}$ & 47.82 & 35.765 & 26.238 \\
\hline ii & Collector ring side (CLRS) & $\mathrm{kW}$ & 45.294 & 33.929 & 25.149 \\
\hline iii & Total seal oil losses & $\mathrm{kW}$ & 93.118 & 69.694 & 51.387 \\
\hline $\mathrm{D}$ & Losses by radiation and convention & $\mathrm{kW}$ & 18.069 & 15.808 & 14.190 \\
\hline E & Total losses & $\mathrm{kW}$ & 3200.51 & 2479.448 & 1612.442 \\
\hline $\mathrm{F}$ & Turbo-generator shaft power input & $\mathrm{kW}$ & $2,222,000.51$ & $166,479.448$ & $111,612.442$ \\
\hline G & Generator efficiency & $\%$ & 98.56 & 98.56 & 98.55 \\
\hline
\end{tabular}

Source: PHCN, Egbin Design Manual [16].

Table 9. Heat balance for unit 6 .

\begin{tabular}{|c|c|c|c|c|c|}
\hline \multirow{2}{*}{$\begin{array}{c}\text { S/N } \\
1\end{array}$} & \multirow[t]{2}{*}{ Item } & \multirow{2}{*}{$\begin{array}{l}\text { Unit } \\
\text { MW }\end{array}$} & \multicolumn{3}{|c|}{ Operating conditions (losses) } \\
\hline & & & 220 & 166 & 110 \\
\hline & Load & $\%$ & 100 & 75.45 & 50 \\
\hline A & Bearing oil losses & & & & \\
\hline i & Turbine side (TBS) & $\mathrm{kW}$ & 61.39 & 42.266 & 27.209 \\
\hline \multirow[t]{2}{*}{ ii } & Collector ring side (CLRS) & $\mathrm{kW}$ & 54.173 & 36.228 & 22.262 \\
\hline & Total bearing losses & $\mathrm{kW}$ & 115.569 & 78.494 & 49.147 \\
\hline B & Losses through $\mathrm{H}_{2}$ coolers & & & & \\
\hline $\mathrm{i}$ & Cooler A & $\mathrm{kW}$ & 789.998 & 557.269 & 419.404 \\
\hline ii & Cooler B & $\mathrm{kW}$ & 743.193 & 596.761 & 419.676 \\
\hline iii & Cooler C & $\mathrm{kW}$ & 788.933 & 557.270 & 452.836 \\
\hline \multirow[t]{2}{*}{ iv } & Cooler D & $\mathrm{kW}$ & 742.524 & 556.069 & 381.125 \\
\hline & Total cooler losses & $\mathrm{kW}$ & 3064.649 & 2267.340 & 1673.041 \\
\hline \multirow[t]{4}{*}{$\mathrm{C}$} & Losses through seal oil & & & & \\
\hline & Turbine side (TBS) & $\mathrm{kW}$ & 45.644 & 36.905 & 26.071 \\
\hline & Collector ring side (CLRS) & $\mathrm{kW}$ & 43.959 & 35.983 & 23.836 \\
\hline & Total losses in seal oil & $\mathrm{kW}$ & 89.603 & 72.888 & 49.907 \\
\hline $\mathrm{D}$ & Losses by radiation and convention & $\mathrm{kW}$ & 18.067 & 16.162 & 12.955 \\
\hline $\mathrm{E}$ & Total losses & & 3287.888 & 2434.888 & 1785.05 \\
\hline $\mathrm{F}$ & Turbo-generator shaft power input & $\mathrm{kW}$ & $223,287.88$ & $168,434.940$ & $111,785.374$ \\
\hline G & Generator efficiency & $\%$ & 98.57 & 98.55 & 98.40 \\
\hline
\end{tabular}




\subsection{Summary of Results}

Table 10 contains the summary of the results of the efficiencies of the turbo-generators at various rated load conditions for the heat balance test process and the guaranteed test.

\section{Analysis of Results}

Tables 7-9 indicate the test result of the efficiencies of the turbo-generators. For unit 1, the efficiency is $98.60 \%$, $98.60 \%$ and $98.42 \%$ at $99.55 \%, 74.09 \%$ and $50.45 \%$ percent load respectively. For unit 4 the efficiency is $98.56 \%, 98.56 \%$ and $98.55 \%$ at $99.55 \%, 74.54 \%$ and $50 \%$ load respectively. While for unit 6 the efficiency is $98.57 \%, 98.55 \%$ and $98.40 \%$ at $100 \%, 75.45 \%$ and $50 \%$ load respectively.

In comparison, the result of the present test shows a very good correlation with the guaranteed efficiency test result of the turbo generator using the work test process. This is represented in Figure 2, which is a plot of efficiency versus load for the various units and test processes. This agreement in the test result was established in spite of the fact that different approaches were used in both test processes. The slight difference in the test result for the various units may be due to the difference in procedure.

Tables 7-9 and Figure 2 show that there is consistency of result between the efficiency values for the various units using the heat balance test process in the efficiency test. Thus, there is no significant variation of the efficiency value between the units as they fall within the same range. The plots of efficiency versus load for the various units follow the same pattern. Plots of load versus losses for the various plants follow the same pattern. This is represented in Figure 3.

Consequently, it is established that the test result are accurate and reliable. It could also be inferred that with good maintenance culture the test result from one unit could positively reflect the test results for other units. A close examination of Figure 2 and Figure 3 show that both the efficiency and losses from the turbo-generator cooling system increases with increase in load. From Figure 4 it can be deduced that the efficiency of the cooling system depend to a large extent on the effectiveness of the hydrogen cooling system since over $93 \%$ of the

Table 10. Summary of test results.

\begin{tabular}{|c|c|c|c|c|c|}
\hline & & Unit 1 & Unit 4 & Unit 6 & \\
\hline \multirow{6}{*}{ Heat balance test process } & Eff. (\%) & 98.6 & 98.6 & 98.42 & \\
\hline & Load (kW) & 219 & 163 & 111 & \\
\hline & Eff. (\%) & 98.56 & 98.56 & 98.55 & \\
\hline & Load (kW) & 219 & 164 & 110 & \\
\hline & Eff. (\%) & 98.57 & 98.55 & 98.4 & \\
\hline & Load (kW) & 220 & 166 & 110 & \\
\hline \multirow{2}{*}{$\begin{array}{c}\text { Guaranteed } \\
\text { test }\end{array}$} & Eff. (\%) & 98.73 & 98.71 & 98.54 & 97.71 \\
\hline & Load (kW) & 220 & 165 & 110 & 55 \\
\hline
\end{tabular}

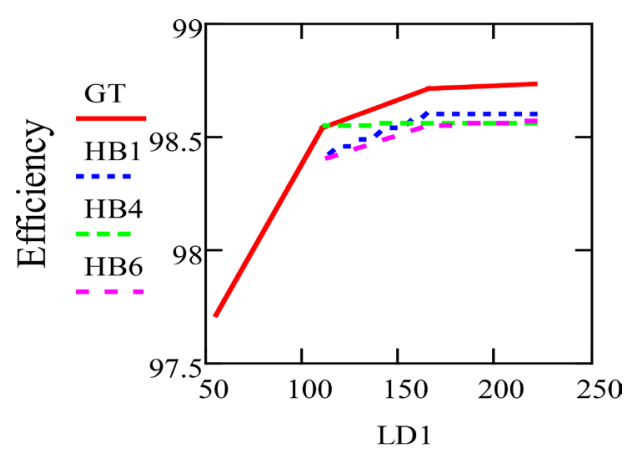

Load $(\mathrm{kW})$

GT: Guaranteed Test, HB: Heat Balance Test, LDI: Load

Figure 2. Plot of efficiency versus load for heat balance and guaranteed test results. 


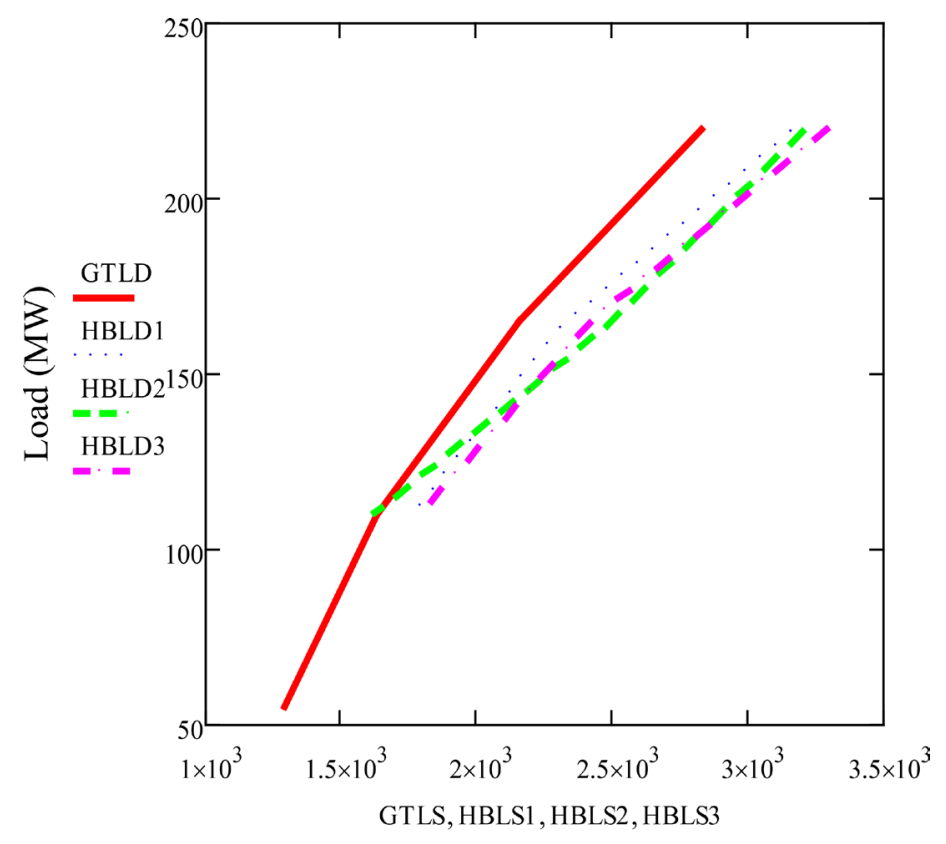

Losses (kW)

GTLD: Guaranteed Test Load, HBLD: Heat Balance Test Load, GTLS: Guaranteed Test Losses, HBLS: Heat Balance Test Losses.

Figure 3. Plots of load versus losses for heat balance and guaranteed test results.

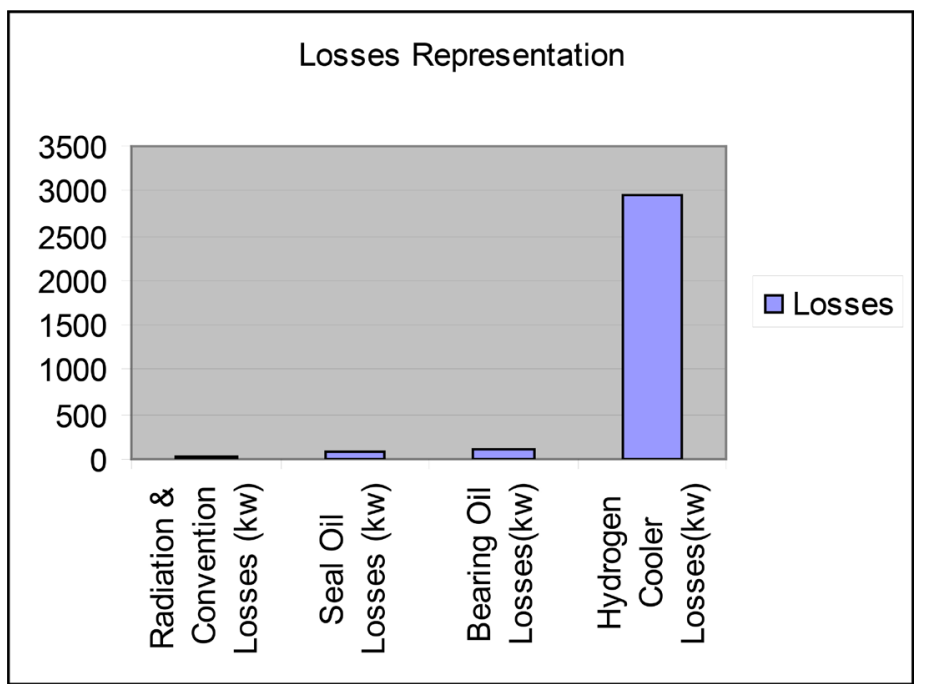

Figure 4. Chart of losses for unit 1.

heat losses in the turbo-generator is removed through this medium. An ineffective hydrogen cooling system will lead to the accumulation of heat in the turbo-generator, raising the temperature of parts and component to levels which could easily lead to their deterioration, breakdown and distortion of shapes. Thus, the efficiency, durability and availability depend on the efficiency of the hydrogen cooling system.

From the tables (Tables 7-9) of flow rates and temperatures readings on the supervisory equipment on the turbo-generators for the same item for corresponding loads, the range of the values of the readings are the same and show slight difference to two places of decimal. This is a pointer to the uniformity of the readings and the accuracy of the test process.

Table 11 shows a summary of losses for turbo-generator unit 1 . 
Table 11. Summary of losses for unit 1.

\begin{tabular}{cc}
\hline Radiation \& convention losses $(\mathrm{kW})$ & 18.82 \\
Seal oil losses $(\mathrm{kW})$ & 89.15 \\
Bearing oil losses $(\mathrm{kW})$ & 104.46 \\
Hydrogen cooler losses $(\mathrm{kW})$ & 2951.91 \\
\hline
\end{tabular}

\section{Conclusions}

The heat balance test to determine the efficiency of the turbo-generators was done by carrying out an energy balance on the machine systems and the efficiency determined from the ratio of output power to input power to the generator shaft. The efficiency results for unit I were $98.60 \%$ at $100 \%$ load, $98.60 \%$ at $75 \%$ load, $98.42 \%$ at $50 \%$, unit 4 had $98.56 \%$ at $100 \%$ load, $98.56 \%$ at $75 \%$ load and $98.55 \%$ at $50 \%$. While those of unit 6 were $98.57 \%$ at $100 \%$ load, $98.55 \%$ at $75 \%$ load and $98.40 \%$ at $50 \%$ load.

Careful observation of the results shows that there exist very good harmony and consistency between the test results using the heat balance test process for the three units. Thus, there is no significant variation of the efficiency value between the units as they fall within the same range. The plots of efficiency versus load for the various units follow the same pattern. Plots of load versus losses for the various plants follow the same pattern also. This high degree of harmony and consistency is also established between the results of the heat balance test results and guaranteed test results. Thus, parallel correlation is hereby established for the three units of turbogenerators in the determination of their efficiencies using the heat balance test process. The heat balance test process is an effective tool to monitor turbo-generator's performance and efficiency profile which serve as a good basis to indicate deviation and deterioration in plants performance.

\section{Acknowledgements}

The authors acknowledged the invaluable assistance of the management of Egbin Thermal Station, Lagos and Mechanical Engineering Department, University of Benin, Benin City for their cooperation during the study. Special appreciation goes to Prof L, A. Salami for his support and counseling in the study.

\section{References}

[1] Hattori, K., Ide, K., Goto, F., Semba, A. and Watanabe, T. (2002) Sophisticated Design of Turbine Generator with Inner Cooler Ventilation System. Hitachi Review, 51, 148-152.

[2] Andreev, A.M., Azizov, A.S., Bezborodov, A.V., Papkov, A.A. and Pak, V.M. (2011) Investigation of Possibility of Developing High Efficiency Conducting System of Electrical Insulation for Turbogenerators with Air and Hydrogen Cooling. Russian Journals of Electrical Engineers, 82, 180-183. http://dx.doi.org/10.3103/S106837121104002X

[3] Nag, P.K. (2001) Power Plant Engineering. 2nd Edition, McGraw Hill, New Delhi.

[4] Kostenko, M. and Piotrovsky, L. (1977) Electrical Machines: Alternating Current Machines. MIR Publisher, Moscow.

[5] Muramatsu, S., Hattori, K., Takahashi, K., Nakahara, A. and Iwashige, K. (2005) Development of 250-MVA AirCooled Turbine Generator. Hitachi Review, 54, 121-125.

[6] Kakimoto, T., Hattori, K., Fugane, K., Mae, H., Kazuhiko, T.K. and Iwashige, K. (2008) High Efficiency and Low Noise Air-Cooled Turbine Generator “GH1550A”. Hitachi Review, 57, $285-291$.

[7] Higgins, R.L. (1995) Maintenance Engineering Handbook. 5th Edition, McGraw Hill, New York.

[8] Gurevic, E.I., Lyamin, A.A. and Shelemba, I.S. (2010) Measurement of the Temperature of a Stator Winding with Fibre Optics Sensors Temperature in Bench Test of a Turbogenerator. Power Technology and Engineering, 44, 249-245.

[9] Hattori, K., Okabe, H., Ide, K., Kobashi, K. and Watambe, T. (2004) Performance Evaluation of a 250 MVA Class Air Cooled Turbogenerator. CIGRE Session, 1-6.

[10] Okabe, H., Onoda, M., Hatori, K., Watambe, T., Morooka, H. and Higashimura, Y. (2003) Development and Performance Evaluation of a High-Reliability Turbine Generator. Hitachi Review, 52, 89-95.

[11] Marrin, H. and Klayto, P.E. (1997) Fundamental Electrical Technology. Addison-Wesley, London.

[12] Zubair, A.M. and Olarewaju, S.O. (2014) Production Index of Electricity Generation and Consumption in Nigeria. International Journal of Engineering and Science, 3, 11-17.

[13] Odungide, M. (2014) Power Statistics in Nigeria. Academia. http://www.academia.com 
[14] Audu, M.L., Zubair, J., Anakhuagbon, A. and Ajaino, O.K. (2012) Efficiency Testing of Turbo-Generators: The Heat Balance Approach. International Journal of Engineering Innovations, 4, 94-102.

[15] PHCN (1978) Egbin Power Station Operation. Manual Vol. 4, Generator and Auxiliaries.

[16] PHCN (1978) Egbin Thermal Station Design Manual. 